\title{
MORE ACTIVE PARTICIPATION IN VOLUNTARY EXERCISE OF OLDER USERS OF INFORMATION AND COMMUNICATIVE TECHNOLOGY EVEN DURING THE COVID-19 PANDEMIC, INDEPENDENT OF FRAILTY STATUS
}

\author{
S. SATAKE ${ }^{1,2}$, K. KINOSHITA ${ }^{1,3}$, H. ARAI ${ }^{4}$ \\ 1. Section of Frailty Prevention, Department of Frailty Research, Center for Gerontology and Social Science, National Center for Geriatrics and Gerontology, Japan; 2. Department of \\ Geriatric Medicine, Hospital, National Center for Geriatrics and Gerontology, Japan; 3. Department of Epidemiology of Aging, Center for Gerontology and Social Science, National \\ Center for Geriatrics and Gerontology, Japan; 4. President, National Center for Geriatrics and Gerontology, Japan. Corresponding author: Shosuke Satake, MD, PhD, Section of Frailty \\ Prevention, Department of Frailty Research, Center for Gerontology and Social Science, National Center for Geriatrics and Gerontology, 7-430, Morioka-cho, Obu, Aichi 474-8511, \\ Japan, Phone: +81-562-46-2311, FAX: +81-562-44-8518, e-mail address: satakes@ncgg.go.jp
}

\begin{abstract}
Objectives: The aim of this study was to examine whether older users of information and communicative technology (ICT) participate in active behaviors, such as voluntary exercise, to maintain health, even under community containment to suppress the coronavirus disease 2019 (COVID-19) pandemic. Design: Cross-sectional study. Setting: Community setting. Participants: Independent community-dwelling older residents aged $\geq 75$ years. Measurements: The municipality sent the Kihon Checklist (KCL) and Simplified Nutritional Appetite Questionnaire (SNAQ) with several questions on lifestyle and health conditions under social restriction. Results: Among 3199 responders (72.1\%), we analyzed the data of 2304 residents who provided complete answers to the KCL and SNAQ and on ICT use, voluntary exercise, polypharmacy, and families. The mean age was 79.7 years $(51.3 \%$ male). The percentages of frailty assessed by the KCL, voluntary exercise, and low SNAQ scores $(\leq 14)$ were $16.0 \%, 61.4 \%$, and $43.8 \%$ in 808 ICT users and $30.3 \%, 47.2 \%$, and $54.1 \%$ in 1496 ICT non-users, respectively $(\mathrm{p}<0.001)$. ICT use was significantly associated with voluntary exercise even during social restriction, independent of age, sex, polypharmacy, low SNAQ scores, and frailty status (odds ratio, 1.503; 95\% confidential interval, 1.246-1.813). Conclusion: Older ICT users are more active to maintain health even during social restriction, independent of frailty status.
\end{abstract}

Key words: Information and communicative technology (ICT), frailty status, voluntary exercise, social restriction, Coronavirus disease-19 (COVID-19).

\section{Introduction}

During the coronavirus disease 2019 (COVID-19) pandemic, many people have been affected by the disease. In many countries, physical distancing and community containment including lockdown have been taken as a countermeasure to suppress the pandemic (1). In Japan, a state of emergency was issued on April 7, 2020, calling for restrictions on interaction with others to prevent COVID-19 infection. Most Japanese citizens responded to government requests and refrained from social interactions (2). As a result, the first wave of the COVID19 pandemic was suppressed to some extent and the state of emergency was lifted on May 25, 2020.

Because such social restrictions can bring about a society with poor connections and low activity, older individuals, especially those who are frail, tend to lack social connections and social support networks and have lower life satisfaction $(3,4)$, are encouraged to take positive actions to maintain their health and connectedness with others while maintaining physical distance. In this sense, information and communication technology (ICT) use can be a helpful strategy to maintain the health of older adults. Moreover, recent studies support this idea because they indicated that ICT use can enhance the psychological well-being of older adults $(5,6)$.

However, it is expected that few older individuals, especially those who are frail, will benefit from it because they are more likely to hold negative opinions regarding the usefulness and usability of mobile ICT (7). There are few reports showing that the use of ICT is associated with frailty status and positive behavior for maintaining physical activity in communitydwelling independent older adults.

Therefore, we conducted a survey to clarify the associations of ICT use with frailty status and physical activity in an independent community-dwelling older population aged $\geq 75$ years during social restriction by the COVID-19 pandemic.

\section{Methods}

\section{Design and subjects}

Of all senior residents aged $\geq 75$ years in Higashi-ura Town on April 1st, 2020, the municipal government identified 4436 independent older residents who were not classified as requiring long-term care insurance services. The government sent a questionnaire to the resident regarding their lifestyle and health condition under social restriction in addition to the Kihon Checklist (KCL) and Simplified Nutritional Appetite Questionnaire (SNAQ) after lifting the state of emergency and asked them to return the questionnaires after answering all questions. We analyzed those who answered all the questions completely. The Ethics Committee of the National Center 
Table 1

Characteristics of ICT user and non-user

\begin{tabular}{|c|c|c|c|c|}
\hline Items & $\begin{array}{c}\text { Total } \\
(n=2304)\end{array}$ & $\begin{array}{l}\text { Non User } \\
(n=1496)\end{array}$ & $\begin{array}{c}\text { User } \\
(n=808)\end{array}$ & p value \\
\hline Age (yrs) & $79.7 \pm 3.9$ & $80.2 \pm 4.1$ & $78.7 \pm 3.3$ & $<0.001$ \\
\hline Men: n (\%) & $1181(51.3)$ & $646(43.2)$ & $535(66.2)$ & $<0.001$ \\
\hline $\mathrm{BMI}\left(\mathrm{kg} / \mathrm{m}^{2}\right)$ & $22.7 \pm 3.1$ & $22.5 \pm 3.3$ & $23.0 \pm 2.9$ & $<0.001$ \\
\hline Living alone: $\mathrm{n}(\%)$ & $349(15.1)$ & $245(16.4)$ & $104(12.9)$ & 0.028 \\
\hline Polypharmacy: n (\%) & $628(27.3)$ & $408(27.3)$ & $220(27.2)$ & n.s. \\
\hline Voluntary exercise during a state of emergency: $\mathrm{n}(\%)$ & $1202(52.2)$ & $706(47.2)$ & $496(61.4)$ & $<0.001$ \\
\hline Simplified Nutritional Appetite Questionnaire: Median [Interquartile range] & $14[13-16]$ & $14[13-16]$ & $15[14-16]$ & $<0.001$ \\
\hline$-\mathrm{SNAQ} \leq 14$ & $1164(50.5)$ & $810(54.1)$ & $354(43.8)$ & $<0.001$ \\
\hline Total Kihon Checklist Score: Median [Interquartile range] & $5[2-8]$ & $5[3-8]$ & $4[2-6]$ & $<0.001$ \\
\hline - Score 0-3 (Robust) & $883(38.3)$ & $489(32.7)$ & $394(48.8)$ & \\
\hline - Score 4-7 (pre-frailty) & $839(36.4)$ & $554(37.0)$ & $285(35.3)$ & $<0.001$ \\
\hline - Score $8+$ (Frailty) & $582(25.3)$ & $453(30.3)$ & $129(16.0)$ & \\
\hline
\end{tabular}

for Geriatrics and Gerontology, Obu, Japan, and Higashi-ura municipal assembly approved the study protocol (\# 1463). Informed consent was obtained by an opt-out method approved by the Personal Information Protection Commission.

\section{Use of Information and Communicative Technology}

ICT use was assessed with the question, "Do you use the internet including e-mail or other communicative applications with computer, smartphone, or tablet devices?" with the following three optional answers: "No, I do not use the internet.", "Yes, I use the internet with help.", and "Yes, I use the internet without help." We considered the latter two answers as ICT users.

\section{Polypharmacy}

Polypharmacy was assessed with the question "How many medicines do you take as prescribed by your doctors?". We considered older residents who answered with "5 and more" as polypharmacy.

Voluntary exercise during social restriction due to a state of emergency

The municipal government assessed active behaviors of residents with the question "Did you do any exercise during social restriction due to a state of emergency?" with "Yes" or "No". We considered any residents who answered with "Yes" as seniors who had been participating in voluntary exercise.

\section{Simplified Nutritional Appetite Questionnaire}

The SNAQ was used to assess nutritional risk with four domains: appetite, feeling of hunger, taste of food, and number of meals per day, with answers ranging from 1 (worst possible) to 5 (8). The total score ranges from 4 to 20 . A prior validation study suggested a cutoff of $\leq 14$ to predict undernutrition and involuntary body weight loss (8).

\section{Kihon Checklist Questionnaire}

The KCL is a simple yes/no questionnaire that assesses multiple aspects of function in daily living, such as instrumental activities of daily living (ADL), social ADL, physical activities and falls, nutrition status, oral functions, outdoor activities, cognitive function, and mood (9). Difficulty with any question was counted as a score in the KCL, with a higher score in each domain of the checklist indicating a higher risk of requiring support or care in that domain.

\section{Definition of frailty}

Frailty status was defined using the total KCL score. We considered older residents with a total KCL score of $0-3$ as robust, $4-7$ as pre-frail, and $8+$ as frail, based on our previous study $(9,10)$.

\section{Statistical analysis}

A chi-squared test was used for categorical classification, and Mann-Whitney test and unpaired Student's t-test were used to analyze differences in non-normal distribution and normal distribution, respectively. Logistic regression analysis was used to estimate the odds ratios (ORs) and $95 \%$ confidence intervals (CIs) for the associations between voluntary exercise and ICT use, living alone, polypharmacy, poor SNAQ score, and frailty status, adjusted for age and sex. All analyses were conducted using StatView v.J-5.0 software (SAS Institute Japan Ltd., Tokyo, Japan). P-values $<0.05$ were considered statistically significant. 
OLDER ICT USERS AND VOLUNTARY EXERCISE

Table 2

Voluntary exercise during social restriction due to a state of emergency

\begin{tabular}{|c|c|c|c|c|c|c|}
\hline \multirow{2}{*}{$\begin{array}{l}\text { Variables } \\
\text { ICT use }\end{array}$} & \multicolumn{2}{|c|}{ Model 1} & \multicolumn{2}{|c|}{ Model 2} & \multicolumn{2}{|c|}{ Model 3} \\
\hline & 1.709 & $1.426-2.050$ & 1.542 & $1.280-1.857$ & 1.503 & $1.246-1.813$ \\
\hline Living alone & 1.253 & $0.991-1.585$ & - & - & - & - \\
\hline Polypharmacy & 0.799 & $0.663-0.962$ & 0.896 & $0.738-1.087$ & 0.920 & $0.758-1.118$ \\
\hline poor SNAQ score $(\leq 14)$ & 0.634 & $0.537-0.748$ & 0.717 & $0.604-0.852$ & 0.737 & $0.620-0.875$ \\
\hline Frailty $(\mathrm{KCL} \geq 8)$ & 0.380 & $0.311-0.464$ & 0.426 & $0.347-0.523$ & - & - \\
\hline \multicolumn{7}{|l|}{ Frailty Status } \\
\hline - Robust (KCL 0-3) & 1 & & & & 1 & - \\
\hline - Pre-frailty (KCL 4-7) & 0.649 & $0.535-0.788$ & - & & 0.695 & $0.571-0.846$ \\
\hline - Frailty $(\mathrm{KCL} \geq 8)$ & 0.304 & $0.243-0.381$ & - & & 0.350 & $0.277-0.441$ \\
\hline
\end{tabular}

Model 1: adjusted for age and sex; Model 2 and 3: all variables were entered into the models at the same time along with age and sex

\section{Results}

Among 4436 senior residents, 3199 seniors (72.1\%) responded. We analyzed the data of 2304 seniors (51.9\%) who filled out their questionnaires completely.

The mean age of participants was 79.7 years, and $51.3 \%$ were males. The number of ICT users and non-users was 808 (35.1\%) and 1496 (64.9\%) residents, respectively. ICT users were slightly younger and had lower malnutrition risk than non-users, but showed the same percentage of polypharmacy as non-users. ICT users were engaged in voluntary exercise during social restriction more frequently $(61.4 \%)$ than non-users $(47.2 \%)$. In addition, the prevalence of frail older adults was $30.3 \%$ and $16.0 \%$ among ICT non-users and users, respectively.

In order to determine the benefit of ICT use on healthy behavior, we analyzed the association of ICT use with voluntary exercise during social restriction in a state of emergency. In logistic regression analyses, ICT use was significantly associated with voluntary exercise during social restriction, adjusted for age, sex, polypharmacy, low SNAQ scores, and frailty status (Table 2).

\section{Discussion}

In this study, we found a significant association between ICT use and positive physical activity even during social restriction due to the COVID-19 pandemic, independent of age, sex, polypharmacy, malnutrition risk, and frailty in communitydwelling older adults aged $\geq 75$ years.

To prevent COVID-19 infection, which has become a significant public health crisis worldwide since December 2019, measures such as minimizing social interaction and promoting physical distancing were taken, but these measures narrow the range of activities and can result in a sedentary lifestyle that causes low physical activity in older adults, especially those who are frail.

Yamada et al. reported that the restriction of social interaction due to the state of emergency during the first wave of the COVID-19 pandemic in Japan decreased the activity time of older adults by $30-35 \%$, independent of frailty status (11). Moreover, they showed that while robust and pre-frail older adults had more physical activity time after the state of emergency was lifted, frail older people who were living alone and lacked social participation did not recover their activity time (12).

From the perspective of continuing social interaction while maintaining social distance, ICT use can help collect information on maintaining health and connections with other people. Therefore, frail older adults who tend to have poor physical function and depressive mood are encouraged to use such technology. However, the rate of ICT usage in frail older adults is reported to be low as they have negative opinions on the usefulness and usability of ICT compared with robust older adults (7). In this study, we also observed that frail older adults used ICT devices less often than robust and pre-frail older adults.

Recently, several studies have reported the benefit of ICT use in older populations. Fang et al. investigated whether ICT use was a potential vehicle for improving the psychological well-being of older adults (5). They concluded that ICT use can potentially enhance the psychological well-being of older adults aged $\geq 75$ years by facilitating their contact with family members. Sims et al. reported that more frequent ICT device and application usage was related to higher levels of psychological well-being among older adults, which was mediated by their motivation to use technology (6). They also showed that ICT use to connect with family and friends was associated with greater life satisfaction, less feelings of loneliness, and higher attainment; whereas learning new information was associated with better subjective health and fewer functional limitations. We found that more ICT users participated in physical activities to maintain their health during social restriction even under the state of emergency compared with ICT non-users. This positive behavior was significantly 


\section{THE JOURNAL OF NUTRITION, HEALTH \& AGING}

associated with ICT use after controlling for age, sex, living alone, polypharmacy, malnutrition risk, and frailty. These results were supported by previous reports on ICT use and health literacy $(6,13)$.

This study has several limitations. First, we did not assess the education levels and economic status of participants, which can influence the use of ICT. Second, we could not confirm their objective health status, such as diseases, types of drugs, objective physical function and mental status, and actual physical activity. Third, we cannot determine whether the significant association between ICT use and voluntary exercise is due to the fact that active older adults use ICT or because ICT use enhances positive behavior to maintain health. Fourth, it is unclear whether voluntary exercise will be encouraged by starting to use ICT after the age of 75 , as we did not ask the participants when they started using ICT. Further intervention studies are needed in the future.

In conclusion, we found a significant association between ICT use and voluntary exercise during the restriction of social interactions to suppress COVID-19 infection, independent of age, sex, polypharmacy, malnutrition risk, and frailty status in community-dwelling residents aged $\geq 75$ years.

Funding: This study was supported by the Research Fund for Longevity Science (30-6) from the National Center for Geriatrics and Gerontology, Japan. The sponsor had no role in the design and conduct of the study; in the collection, analysis, and interpretation of the manuscript; or in the review or approval of the manuscript.

Acknowledgements: The authors would like to thank Noriko Ogama for screening, sorting, and organizing the data, and Tomoko Nakajima and Kyoko Yoshinaga for their secretarial assistance. We would also like to thank older residents in the Higashi-ura town who cooperated in the questionnaire, and all the staffs of Higashi-ura City Hall.

\section{Conflicts of interests: None.}

Author's contributions: Shosuke Satake conceived the study design, performed the data analysis, interpreted the results, drafted the initial manuscript, and had primary responsibility for the final content. Kaori Kinoshita conceived the study design, interpreted the results, and performed the data analysis. Hidenori Arai interpreted the results, contributed to discussions, and supervised the study. All authors critically revised the manuscript for intellectual content and approved the final manuscript.
Ethical standards: The ethics committee approved the study.

\section{References}

1. Wilder-Smith A, Freedman DO. Isolation, quarantine, social distancing and community containment: pivotal role for old-style public health measures in the novel coronavirus (2019-nCoV) outbreak. J Travel Med 2020; 27(2).

2. Lim WS, Liang CK, Assantachai P, et al. COVID-19 and older people in Asia: Asian Working Group for Sarcopenia calls to actions. Geriatr Gerontol Int. 2020; 20(6):547 558.

3. Jeon G-S, Jang S-N, Park S. Social support, social network, and frailty in Korean elderly. J Korean Geriatr Soc 2012;16(2):84-94

4. Yang F, Gu D, Mitnitski A. Frailty and life satisfaction in Shanghai older adults: the roles of age and social vulnerability. Arch Gerontol Geriatr 2016;67:68-73.

5. Fang Y, Chau AKC, Wong A, Fung HH, Woo J. Information and communicative technology use enhances psychological well-being of older adults: the roles of age, social connectedness, and frailty status. Aging Ment Health 2018;22(11):1516-1524

6. Sims T, Reed AE, Carr DC. Information and communication technology use is related to higher well-being among the oldest-old. J Gerontol B Psychol Sci Soc Sci 2017;72(5):761-770.

7. Keränen NS, Kangas M, Immonen M, Similä H, Enwald H, Korpelainen R, Jämsä T. Use of information and communication technologies among older people with and without frailty: a population-based survey. J Med Internet Res 2017;19(2):e29.

8. Wilson MM, Thomas DR, Rubenstein LZ, Chibnall JT, Anderson S, Baxi A, Diebold MR, Morley JE. Appetite assessment: simple appetite questionnaire predicts weight loss in community-dwelling adults and nursing home residents. Am J Clin Nutr 2005;82(5):1074-1081.

9. Satake S, Senda K, Hong YJ, Miura H, Endo H, Sakurai T, Kondo I, Toba K. Validity of the Kihon Checklist for assessing frailty status. Geriatr Gerontol Int 2016;16(6):709-715.

10. Satake S, Shimokata H, Senda K, Kondo I, Toba K. Validity of total Kihon Checklist score for predicting the incidence of 3-year dependency and mortality in a communitydwelling older population. J Am Med Dir Assoc 2017;18(6):552.e1-552.e6.

11. Yamada M, Kimura Y, Ishiyama D, Otobe Y, Suzuki M, Koyama S, Kikuchi T, Kusumi H, Arai H. Effect of the COVID-19 epidemic on physical activity in community-dwelling older adults in Japan: a cross-sectional online survey. J Nutr Health Aging 2020;24(9):948-950.

12. Yamada M, Kimura Y, Ishiyama D, Otobe Y, Suzuki M, Koyama S, Kikuchi T, Kusumi H, Arai H. Letter to the editor: recovery of physical activity among older Japanese adults since the first wave of the COVID-19 pandemic. J Nutr Health Aging 2020;24(9):1036-1037.

13. Kobayashi LC, Wardle J, von Wanger C. Internet use, social engagement and health literacy decline during ageing in a longitudinal cohort of older English adults. J Epidemiol Community Health 2015;69(3):278-283. 